\title{
BMJ Open What to measure in biliary atresia research: study protocol for developing a core outcome set
}

\author{
Christopher Maguire (D) , ${ }^{1}$ Zephanie Tyack (1) , ${ }^{2}$ Roy M Kimble, ${ }^{3}$ \\ Bronwyn Ruth Griffin (1) ,,5 Helen Joanna Yesberg, ${ }^{6}$ Fang Ming Choo, ${ }^{6}$ \\ Craig Antony McBride (i) 7,8
}

\begin{abstract}
To cite: Maguire C, Tyack Z, Kimble RM, et al. What to measure in biliary atresia research: study protocol for developing a core outcome set. BMJ Open 2021;11:e047224. doi:10.1136/ bmjopen-2020-047224
\end{abstract}

- Prepublication history and additional supplemental material for this paper are available online. To view these files, please visit the journal online (http://dx.doi.org/10.1136/ bmjopen-2020-047224).

Received 22 March 2021 Accepted 26 July 2021

\section{A Check for updates}

(C) Author(s) (or their employer(s)) 2021. Re-use permitted under CC BY-NC. No commercial re-use. See rights and permissions. Published by BMJ.

For numbered affiliations see end of article.

\section{Correspondence to} Dr Christopher Maguire; christopher.r.maguire@gmail. com

\section{ABSTRACT}

Aim Extrahepatic biliary atresia is a rare disorder. This creates challenges in the quality and quantity of research conducted. This issue is exacerbated by the potential heterogeneity in the reported outcomes in research examining the management of biliary atresia. A core outcome set is required to standardise reporting on the management of biliary atresia in research, facilitate systematic reviews that include outcomes of greatest importance to patients and clinicians, and to evaluate the quality of the existing evidence base on the management of biliary atresia.

Methods A list of all potential outcomes will be developed through a systematic review of the literature. This list will be refined through a three-stage Delphi approach, involving key stakeholders in the management of biliary atresia. This will include patients and their parents, clinicians, nurses and allied health professionals. In this way, outcomes will be prioritised into a set of consensus core outcomes.

Conclusion The development of a core outcome set in biliary atresia management is needed to guide future research and assist in evaluating the quality of existing research.

Ethics and dissemination Ethical approval has been granted by the Children's Health Queensland Hospital and Health Service Human Research Ethics Committee (HREC), Ref: $H R E C / 20 / Q C H Q / 62448$. Results of the study will be published in an open access format.

\section{INTRODUCTION}

Biliary Atresia is a rare, progressive fibroobliterative condition of the extrahepatic biliary tree, occurring in approximately one in every 17000 births. ${ }^{1}$ As opposed to being one disease process, the clinical description of biliary atresia likely represents a phenotype of pathology that is characterised by extrahepatic biliary obliteration and variably compromised intrahepatic ductules. ${ }^{2}$ The rarity of this condition and the need for quality data that assess the relationship between clinical interventions and outcomes have led to the call for the development of national databases. These resources aim to

\section{Strengths and limitations of this study}

- This protocol describes the first international consensus effort to develop a core outcome set (COS) for use in biliary atresia research and thereby inform the ongoing development and management of the Biliary Atresia Registry of Australia and New Zealand

- The systematic review and COS development methodology used complies with Core Outcome Measures in Effectiveness and Preferred Reporting Items for Systematic Reviews and Meta-Analyses guidelines.

- The study engages multiple stakeholder perspectives through a Delphi process that includes patients, clinicians, nurses and allied health professionals from multiple health services.

- This project is limited by a lack of robust guidelines for, and evidence regarding, best practice in stakeholder composition and weighting in COS development.

- The regional focus of this paper also represents a limitation in its applicability to the international community.

facilitate collaboration in research and to broaden outcome reporting. ${ }^{3}$ However, this raises the question of what outcomes should be reported, and in what order of importance. Despite the identification of inconsistency in outcome reporting among biliary atresia publications, up until now, this deficiency has not been rectified by the development of a core outcome set (COS) for biliary atresia research. ${ }^{4}$ This problem is not unique to biliary atresia. COS development has been increasingly recognised as an important adjunct to the development of all research methodology. ${ }^{5-8}$ The rationale for this is the need to improve the comparability of data sets across similar trials or databases, reduce selective outcome reporting, and increase the relevance of results to key stakeholders from trials and systematic reviews. ${ }^{9-12} \mathrm{COS}$ 
set development has been guided by the Core Outcome Measures in Effectiveness Trials (COMET) initiative, and further refined by the consensus-based standards for the selection of health measurement instruments (COSMIN) initiative. ${ }^{13}$ This study aims to develop a COS for biliary atresia research in Australia and New Zealand and, in doing so, inform the ongoing development and management of the Biliary Atresia Registry of Australia and New Zealand. This has the benefit of bringing patient and family views into the discussion of what to report and prioritise in biliary atresia research. Where data on an outcome are lacking and families and professionals see that outcome as important, research can then be directed regionally towards addressing those shortfalls.

\section{METHODS AND ANALYSIS}

The methodology of this study has been developed in accordance with the principles outlined in the Core Outcome Set-Standards for Development (COSSTAD) ${ }^{9}$ The specific aims of this study are: first, to develop a comprehensive list of clinical outcomes in biliary atresia management within the extant literature; second, to prioritise these outcomes from a patient and clinician perspective; and third, to document a consensus on a minimum set of relevant outcomes for biliary atresia management. A mixed methods design will be employed in order to adhere to the best practice in COS development outlined in the COS-STAD framework. This will include: (1) the identification of existing reported outcomes through a systematic review of the literature; (2) the conduct of a Delphi survey to determine which outcomes are to be considered in a COS in biliary atresia management; and (3) the conduct of a set of consensus meetings in which a COS is determined. This study has been registered with PROSPERO (CRD42020180133).

\section{Inclusion criteria}

The inclusion criteria for stakeholder participation in this study will be patients aged 16 years or older, parents of patients with biliary atresia of any age, or clinicians, nurses, or allied health professionals who have had a direct input into the management of patients with biliary atresia. The inclusion of patients and patients' parents in the development of this COS is seen as essential to ensure the relevance of outcome measures to those with lived experience of the condition and reflects best practice in COS construction and ultimately the quality of the evidence base. ${ }^{11} 1415$ In doing so, we also hope to gain additional insights into the relative importance of various outcomes from the perspective of those living with the condition.

\section{Exclusion criteria}

The exclusion criteria for stakeholder participation in this study will be patients less than 16 years of age (due to difficulties in ensuring an adequate understanding of the relevant outcome measures, the potential distress caused by asking young patients to consider outcomes related to mortality, and the questionable capacity young children have to participate in an online survey or consensus meeting), those who lack the capacity to consent to participation in surveys, patients under the guardianship of the state (due to difficulties in gaining consent), and those who do not speak or read English. This study will also exclude patients who have had a liver transplant, or their representatives.

\section{Study setting}

Delphi survey participants will be recruited from multiple health services around Australia and internationally.

\section{Scope}

The BA COS systematic review will include all articles that report outcomes in the management of patients with biliary atresia. This includes all systematic reviews, randomised controlled trials, quasi-experimental designs including case-controlled trials, case series, prospective cohort and cross-sectional studies, qualitative studies, mixed methods studies and review articles. Case studies, or case series with fewer than three patients, opinion pieces, surveys, discussion papers and consensus papers will be excluded.

\section{Patient and public involvement}

Inherent to the development of a COS is the aim of highlighting the priorities, experiences and preferences of a condition from patient and clinician perspectives. In this project, patient involvement will form an integral component of the Delphi survey and consensus meetings that will be used to inform the development of the final COS. While patients with biliary atresia have not been involved in the design of this study, the methodology has been built to conform with established international standards in COS development as outlined in COS-STAD. The intent for publication of this project will be to make it open access, and in this way make the findings universally accessible for researchers, clinicians and patients alike.

\section{Phase 1: identification of existing outcome measures Systematic review}

The intent of the review will be to identify a comprehensive list of all reported outcomes in the clinical management of biliary atresia, and to categorise those outcomes into domains. In this way, we hope to summarise any existing heterogeneity of outcome reporting and formulate a consolidated 'outcome list' that can form the basis of the development of a COS. The databases used will include: Embase, PubMed, Cochrane/Central, Scopus, PsychINFO and Cumulated Index to Nursing and Allied Health Literature (CINAHL). Searches have been constructed with the assistance of a medical librarian from the University of Queensland with expertise in conducting systematic review searches. Where possible within the selected databases, Medical Subject Headings, including subheadings, have been preferentially used and augmented with free text (keywords) in order 
to supplement inadequate indexing within $\mathrm{MeSH}$ (or equivalent) databases. Free text terms were selected via consensus of the research team to minimise the potential for missing relevant articles and include: 'biliary atresia,' OR, 'bile duct atresia,' OR, 'hepatoportoenterostomy,' OR 'Kasai portoenterostomy,' OR 'portoenterostomy, hepatic.' Full search strategies for each of the databases along with an explanation of the search construction are provided in online supplemental appendix A.

Using the Cochrane approved systematic review tool Covidence, two reviewers will independently assess and apply the inclusion and exclusion criteria to title and abstract screening of the first 200 papers that are delivered by the search. At the end of this process, the reviewers will meet to examine their level of agreement and address any inconsistencies in the application of the criteria. If less than $80 \%$ agreement is achieved between the reviewers, then a further 200 results will be assessed, and inconsistencies will be discussed. Following this, the two reviewers will independently screen the title and abstracts of the papers. At the end of the screening process, disagreements will be resolved through discussion between the two reviewers or if agreement cannot be reached through the involvement of a third reviewer. Following title and abstract screening, the full texts of included papers will be independently reviewed by the two reviewers.

Outcomes will be defined, as outlined in the COMET handbook, as 'a measurement or observation used to capture and assess the effect of treatment such as assessment of side effects (risk) or effectiveness (benefits)' ${ }^{16}$ These outcomes will be extracted from the relevant articles, using a standardised proforma based on the Cochrane collaboration good practice data collection form, and collated into a 'unique outcome long list'. This process will be completed according to the methodology outlined by Young et al. ${ }^{17}$ As the intent of this process is to develop a comprehensive list of outcome measures in biliary atresia research, no studies will be excluded based on quality. Outcomes that relate to transplantation, or patients with biliary atresia following transplantation will not be considered as these outcomes are beyond the scope of this study.

\section{Phase 2: domain allocation and questionnaire design}

Following the development of a unique outcome long list from the systematic review, each outcome will be, where possible, allocated to a domain that includes similar outcomes. This will be done independently by two researchers (CRM) and (CM), with subsequent discussion to determine disagreement and resolution of appropriate grouping of outcomes into domains, and the name of each domain. These final unique outcomes, and attributed domains, will then be reviewed by a patient representative who will provide feedback regarding clarity and appropriateness of outcome classification. A survey questionnaire will then be developed around these domains to ascertain the respondent's impression regarding the relative importance of each domain to the overall progression of research into biliary atresia. Each question will be designed in conjunction with a small group of stakeholders from both a clinical and patient/ parent background to ensure that it is comprehensible to both stakeholder groups. Where medical terms are used, they will be coupled with plain language or will be defined to avoid confusion by participants.

Phase 3: Delphi survey

Delphi methodology

Utilisation of a Delphi survey to refine a COS has been chosen in accordance with current COS methodology and COMET guidelines. ${ }^{16}{ }^{18-20}$ A survey will be distributed to stakeholders via email and completed anonymously in three rounds. The only identifying data that will be requested are the respondent's 'stakeholder status' (ie, parent, patient, surgeon, physician, etc), age and gender. Within this survey, participants will be asked to prioritise outcome domains according to importance. A Likert scale ranging from 'not that important' (0) to 'critical' (9) will be used to ascertain the participants' impressions of the relative importance of each outcome to the management of biliary atresia. Specifically, participants will be asked to consider the COS from the perspective of "most important in progressing the health and well-being of patients with biliary atresia .' The Delphi survey will include doctors, nurses, allied health professionals, patients (above 16 years of age), and parents. All outcomes from round one will remain in the survey for round two in order to allow participants to reconsider their initial ranking in the context of the response from other respondents. Round two will include outcome ranking analytics that will show respondents how much agreement there was in the initial round. Outcomes that achieve a score of $7-9$ by $50 \%$ of respondents and $1-3$ by less than $15 \%$ of either patients/ parents or professionals after rounds 1 and 2 will be considered for inclusion in round 3. In round 3, essential outcomes will be defined as those that achieve a score of $7-9$ by $70 \%$ or more of respondents and $1-3$ by less than $15 \%$ of respondents will be chosen for progression to the consensus meeting phase.

\section{Sample size and stakeholder group breakdown}

There is no current consensus regarding the optimal participant sample size required to ensure that a robust Delphi survey is conducted within COS development. ${ }^{916}$ While some guidance can be taken from previous similar works such as that implemented by Schmitt et al, in the development of a COS for eczema management in which there were 46 participants, ${ }^{21}$ there is a wide disparity across the international literature. As such a pragmatic approach will be taken, where the number of clinicians involved will represent the maximum number of surgeons or physicians who specialise in biliary atresia management in Australia and New Zealand who are able to participate. Patient and research groups will then be filled to match this number such that all stakeholder groups are equally represented. The research team are aiming for 
approximately 15-20 people in each stakeholder group. While the focus of this study is on creating a regional COS, the authors feel the input of international experts would be valuable, and as such a small number of international participants will be invited to provide an external perspective on the question.

Additionally, while the COS-STAD do not provide a minimum threshold of participants they do outline a minimum requirement in the category of stakeholder groups to be represented. These include: those who will use the COS in research, healthcare professionals and patients or their representatives. ${ }^{9}$ For this study, the stakeholder group breakdown will be as follows: healthcare professionals including surgeons who perform the Kasai procedure, gastroenterologists or hepatologists who manage patients with biliary atresia, and nursing/ allied health staff that have specialised experience in the management of patients with biliary atresia; those who will use the COS in research including clinical and non-clinical academics who have published research specifically on outcomes in biliary atresia management; finally, patients including patients who have been diagnosed with biliary atresia are above the age of 16, and have not had a liver transplant. This latter group will also include patient representatives, specifically parents of patients with biliary atresia (where those patients have not been transplanted). No specific parents' associations or medical specialist associations will be involved.

\section{Data collection and storage}

All information from the survey rounds, conducted during the Delphi process will be conducted through Research Electronic Data Capture (REDCap) secure data collection tool. This tool will be used to both facilitate online surveys and store participants' information. The storage of limited participant information is required in order for them to be contacted for future survey rounds and participation in the consensus meeting stage. As a consequence, a secure data collection software package is required. REDCap is approved by our hospital health service for the purposes of recording and storing patient clinical information and provides a robust package of services that can securely facilitate the management of participant information throughout the Delphi survey rounds.

\section{Phase 4: consensus meeting}

Consensus meetings will be held following the aggregation of data from the initial survey. All participants who completed the survey will be invited to attend. Two separate meetings will be held; the first for patients/ parents, and the second for professionals. Consideration will be given to allowing participants to attend remotely to account for travel limitations and public health requirements. The results of the survey will be presented at the meeting, and attendees will be asked to rate each outcome as 'core' or 'not core.' Voting will continue to occur in an iterative fashion until a twothirds majority is reached regarding each outcome's inclusion or exclusion. The professional meeting will be held following the patient meeting to allow clinicians to consider patient/parent preferences. In the event that an outcome considered 'not core' by one meeting is considered 'core' by another, it will be included in the final core outcome list. In essence, both groups would need to determine that an outcome is not a core outcome for it to be removed from consideration.

\section{Ethics and dissemination}

Ethical approval was obtained from Children's Health Queensland Hospital and Health Service Human Research Ethics Committee (HREC/20/QCHQ/62448). Date of approval 22/07/2020. As a part of ethics approval for this research, a comprehensive consent process has been developed. This has included the development of separate consent information forms for a number of stakeholder groups including parents, patients and clinicians, that are tailored to the particular risks of their involvement. Taking part in the first survey of the Delphi process will be taken as implied consent for the purposes of the research project. Patient confidentiality will be maintained by utilising the REDCap database system which is password protected through institutional login. Results will be disseminated in the form of open access publication in a peer reviewed journal.

\section{Author affiliations}

${ }^{1}$ General Surgery, Queensland Health, Brisbane, Queensland, Australia

${ }^{2}$ Centre for Children's Burns and Trauma Research, University of Queensland, South Brisbane, Queensland, Australia

${ }^{3}$ Lady Cilento Children's Hospital, Centre for Children's Burns \& Trauma Research,

Brisbane, Queensland, Australia

${ }^{4}$ Centre for Children's Burns and Trauma, Queensland University of Technology Faculty of Health, Kelvin Grove, Queensland, Australia

${ }^{5}$ Pegg Leditschke Children's Burns Centre, Children's Health Queensland, South Brisbane, Queensland, Australia

${ }^{6}$ Faculty of Science, University of Queensland, Brisbane, Queensland, Australia

${ }^{7}$ Lady Cilento Children's Hospital, Queensland Health, Brisbane, Queensland, Australia

${ }^{8}$ School of Medicine, University of Queensland, Brisbane, Queensland, Australia

\section{Twitter Zephanie Tyack @tyack_z and Craig Antony McBride @PaedSurg}

Contributors CM is the lead researcher on this project. He is involved in all aspects of the development, drafting and implementation of this methodology. CAM is the primary research supervisor for this project and is involved in the development and review of the methodology as well as editing of the submission. ZT and BRG are associate research supervisors and have been involved in the development and review of the methodology as well as editing of the submission. RK is the lead clinical supervisor of the development and implementation of the methodology outlined. HJY and FMC are research associates who have contributed to the drafting and editing of the manuscript.

Funding The authors have not declared a specific grant for this research from any funding agency in the public, commercial or not-for-profit sectors.

Competing interests None declared.

Patient consent for publication Not required.

Provenance and peer review Not commissioned; externally peer reviewed.

Supplemental material This content has been supplied by the author(s). It has not been vetted by BMJ Publishing Group Limited (BMJ) and may not have been peer-reviewed. Any opinions or recommendations discussed are solely those 
of the author(s) and are not endorsed by BMJ. BMJ disclaims all liability and responsibility arising from any reliance placed on the content. Where the content includes any translated material, BMJ does not warrant the accuracy and reliability of the translations (including but not limited to local regulations, clinical guidelines, terminology, drug names and drug dosages), and is not responsible for any error and/or omissions arising from translation and adaptation or otherwise.

Open access This is an open access article distributed in accordance with the Creative Commons Attribution Non Commercial (CC BY-NC 4.0) license, which permits others to distribute, remix, adapt, build upon this work non-commercially, and license their derivative works on different terms, provided the original work is properly cited, appropriate credit is given, any changes made indicated, and the use is non-commercial. See: http://creativecommons.org/licenses/by-nc/4.0/.

\section{ORCID iDs}

Christopher Maguire http://orcid.org/0000-0002-4179-2089

Zephanie Tyack http://orcid.org/0000-0003-3376-5731

Bronwyn Ruth Griffin http://orcid.org/0000-0002-6182-9125

Craig Antony McBride http://orcid.org/0000-0001-8377-1748

\section{REFERENCES}

1 McKiernan PJ, Baker AJ, Kelly DA. The frequency and outcome of biliary atresia in the UK and ireland. Lancet 2000;355:25-9.

2 Lakshminarayanan B, Davenport M. Biliary atresia: a comprehensive review. J Autoimmun 2016;73:1-9.

3 Verkade HJ, Bezerra JA, Davenport M, et al. Biliary atresia and other cholestatic childhood diseases: advances and future challenges. $J$ Hepatol 2016;65:631-42.

4 Tyraskis A, Parsons C, Davenport M. Glucocorticosteroids for infants with biliary atresia following Kasai portoenterostomy. Cochrane Database Syst Rev 2018;5:CD008735.

5 Clarke M. Standardising outcomes for clinical trials and systematic reviews. Trials 2007;8:39.

6 Clarke M. Standardising outcomes in paediatric clinical trials. PLOS Med 2008;5:e102.

7 Cooney RM, Warren BF, Altman DG, et al. Outcome measurement in clinical trials for ulcerative colitis: towards standardisation. Trials 2007;8:17.
8 Kirkham JJ, Gargon E, Clarke M, et al. Can a core outcome set improve the quality of systematic reviews? - a survey of the Coordinating editors of cochrane review groups. Trials 2013;14:1.

9 Kirkham JJ, Davis K, Altman DG, et al. Core outcome Set-STAndards for development: the COS-STAD recommendations. PLoS Med 2017; 14:e1002447.

10 Gargon E, Gorst SL, Williamson PR. Choosing important health outcomes for comparative effectiveness research: 5th annual update to a systematic review of core outcome sets for research. PLoS One 2019;14:e0225980.

11 Williamson PR, Altman DG, Blazeby JM, et al. Developing core outcome sets for clinical trials: issues to consider 2012;13.

12 Sinha I, Jones L, Smyth RL, et al. A systematic review of studies that aim to determine which outcomes to measure in clinical trials in children. PLOS Med 2008;5:e96-78.

13 Core outcome measures in effectiveness trials. Available: http://www. comet-initiative.org/

14 Sanderson T, Morris M, Calnan M, et al. What outcomes from pharmacologic treatments are important to people with rheumatoid arthritis? creating the basis of a patient core set. Arthritis Care Res 2010;62:640-6.

15 Stewart RJ, Caird J, Oliver K, et al. Patients' and clinicians' research priorities. Health Expect 2011;14:439-48.

16 Williamson PR, Altman DG, Bagley H. The comet Handbook: version 1.0. 18, 2017.

17 Young AE, Brookes ST, Avery KNL, et al. A systematic review of core outcome set development studies demonstrates difficulties in defining unique outcomes. J Clin Epidemiol 2019;115:14-24.

18 Sinha IP, Smyth RL, Williamson PR. Using the Delphi technique to determine which outcomes to measure in clinical trials: recommendations for the future based on a systematic review of existing studies. PLoS Med 2011;8:e1000393.

19 Hasson F, Keeney S, McKenna H. Research guidelines for the Delphi survey technique. J Adv Nurs 2000;32:1008-15.

20 Chia-Chien H, Brian AS. The Delphi technique: making sense of consensus. Practical Assessment 2007;12:1-8

21 Schmitt J, Langan S, Stamm T, et al. Core outcome domains for controlled trials and clinical recordkeeping in eczema: international multiperspective Delphi consensus process. J Invest Dermatol 2011;131:623-30. 